\title{
Early decrease in serum amphiregulin or vascular endothelial growth factor levels predicts sorafenib efficacy in hepatocellular carcinoma
}

\author{
CORINNE GODIN ${ }^{1,2 *}$, SANDRA BODEAU ${ }^{2,3 *}$, ZUZANA SAIDAK $^{1,4}$, CHRISTOPHE LOUANDRE $^{2}$, \\ CATHERINE FRANÇOIS ${ }^{5}$, JEAN-CLAUDE BARBARE ${ }^{6}$, ROMAIN CORIAT $^{7,8}$, \\ ANTOINE GALMICHE ${ }^{1,2}$ and CHLOÉ SAUZAY ${ }^{1,2}$
}

\author{
${ }^{1}$ Equipe CHIMERE, EA 7516, Université de Picardie Jules Verne; ${ }^{2}$ Laboratoire de Biochimie; ${ }^{3}$ Laboratoire de \\ Pharmacologie; ${ }^{4}$ Service d'Oncobiologie Moléculaire, Centre de Biologie Humaine, CHU Sud; ${ }^{5}$ EA4294, Université de \\ Picardie Jules Verne; ${ }^{6}$ Délégation à la Recherche Clinique et à l'Innovation, CHU Sud, 80054 Amiens; \\ ${ }^{7}$ Gastroenterology and Digestive Oncology Unit, Cochin University Hospital, APHP; ${ }^{8}$ Unité Inserm U1016, \\ Université Paris Descartes, Sorbonne Paris Cité, 75006 Paris, France
}

Received June 29, 2018; Accepted November 15, 2018

DOI: $10.3892 / o r .2018 .6922$

\begin{abstract}
Sorafenib is the standard of care for the treatment of advanced hepatocellular carcinoma (HCC). However, identifying secreted biomarkers that predict sorafenib efficacy in all HCC patients remains challenging. It was recently reported that sorafenib interferes with protein homeostasis and inhibits global translation in tumour cells. A likely consequence of this inhibition would be the interruption of autocrine loops. The aim of the present study was to investigate the effect of sorafenib on two growth factors implicated in autocrine loops and HCC tumour invasion: amphiregulin (AREG) and vascular endothelial growth factor (VEGF). ELISA, quantitative polymerase chain reaction analysis, western blotting and a cytokine array were performed on HCC cell lines and the prognostic role of these two biomarkers in HCC patients was evaluated. Serum AREG and VEGF levels were assayed by ELISA in 55 patients with advanced HCC treated with sorafenib. It was observed that sorafenib decreased AREG, VEGF and cytokine expression
\end{abstract}

Correspondence to: Dr Chloé Sauzay, Laboratoire de Biochimie, Centre de Biologie Humaine, Avenue René Laennec, Centre de Biologie Humaine, CHU Sud, 80054 Amiens, France

E-mail: sauzay.chloe@chu-amiens.fr

${ }^{*}$ Contributed equally

Abbreviations: AREG, amphiregulin; EGFR, epidermal growth factor receptor; OS, overall survival; PFS, progression-free survival, UPR, unfolded protein response; VEGF, vascular endothelial growth factor

Key words: amphiregulin, vascular endothelial growth factor, sorafenib, hepatocellular carcinoma, prognostic biomarker, proteostasis at the transcriptional and post-transcriptional levels. All HCC patients in our cohort had detectable concentrations of AREG and VEGF both at baseline and after sorafenib treatment. The decreased serum levels of AREG and VEGF after 15 days of sorafenib treatment were significantly associated with better overall and progression-free survival. The results of the multivariate analysis demonstrated that a decrease in AREG was an independent prognostic indicator of overall survival (hazard ratio, 0.208; 95\% confidence interval, 0.173-0.673; $\mathrm{P}=0.0003$ ). These results suggest that sorafenib inhibits autocrine loops and that early decrease in serum AREG or VEGF levels predicts sorafenib efficacy in HCC patients.

\section{Introduction}

Liver cancer is the fifth most prevalent cancer and the second most frequent cause of cancer-related mortality worldwide (1). Hepatocellular carcinoma (HCC) is the most frequent type of primary liver tumour. The majority of patients are diagnosed at an advanced stage, which explains the poor prognosis of this cancer. Sorafenib is the standard of care for the treatment of advanced HCC. Sorafenib is a multikinase inhibitor directed against the RAF kinases and several receptor tyrosine kinases, such as vascular endothelial growth factor receptor (VEGFR) (2). The efficacy of sorafenib was demonstrated a decade ago in two comparative placebo-controlled trials that showed a benefit in terms of overall survival (OS) $(3,4)$. Recently, lenvatinib demonstrated a survival advantage that was non-inferior to that of sorafenib in an open-label, multicentre, non-inferiority, randomised trial (5). However, sorafenib currently remains the only first-line treatment approved by the Food and Drug Administration for patients with advanced HCC.

There is currently a lack of biomarkers able to predict the efficacy of sorafenib in HCC patients. $\alpha$-Fetoprotein (AFP) is the biomarker most widely used by clinicians for the follow-up of HCC patients treated by sorafenib. However, 
AFP is secreted in only $~ 50 \%$ of HCC patients due to high intratumoural variability $(6,7)$. Among these patients, only $37 \%$ exhibit a significant decrease in their AFP levels following sorafenib treatment (8). Recently, the latest recommendations of the European Association for the Study of the Liver have underlined the suboptimal performance of AFP as a serological test in the surveillance of HCC patients (1). Identifying a biomarker secreted in all patients with HCC that is directly regulated by sorafenib remains a challenge. In a recent study exploring the impact of sorafenib on proteostasis, we reported that sorafenib inhibits global protein biosynthesis and initiation of translation (9). Proteostasis may be defined as the process regulating the production, folding, trafficking and degradation of proteins within the cell, in order to maintain its homeostasis. In HCC cells exposed to sorafenib, this inhibition of translation is expected to largely prevent the synthesis of several biologically active peptides and proteins. Although this putative effect has not been formally examined, a likely consequence of protein synthesis inhibition would be interruption of autocrine loops (10).

Proliferation of HCC cells is dependent on two well-documented autocrine loops composed of VEGF/VEGFR and amphiregulin (AREG)/epidermal growth factor receptor (EGFR) ligands and receptors. VEGFR is a direct target of sorafenib and is known to be an important factor in $\mathrm{HCC}$ growth (11). VEGF is a potent angiogenic factor and is upregulated in several human tumours (12). This autocrine loop plays a crucial role in tumour invasion and aggressiveness (12). Activation of EGFR during hepatocarcinogenesis is not dependent on activating mutations, but rather on autocrine loops involving natural ligands of EGFR, such as AREG (13). AREG is upregulated in HCC and acts as a pro-oncogenic factor (14). This autocrine loop was recently identified as a key determinant of resistance to sorafenib $(15,16)$. The aim of the present study was to evaluate the effect of sorafenib on AREG and VEGF production in two HCC cell lines. In order to study a potential link between AREG and VEGF production and the efficacy of sorafenib, the prognostic impact of each of these factors was investigated in HCC patients.

\section{Materials and methods}

Cell culture and reagents. The human HCC cell lines used in this study (Huh7 and Hep3B, passages between 6 and 8) were obtained from Dr Wychowski (Institut de Biologie de Lille, Lille, France) and were authenticated by profiling short tandem repeats at 16 loci (LGC Standards, Strasbourg, France). The cells were cultured in Dulbecco's modified Eagle's medium supplemented with $10 \%$ fetal calf serum (Jacques Boy Institute S.A., Reims, France), $2 \mathrm{mM}$ glutamine, penicillin and streptomycin. Brefeldin A was purchased from Sigma-Aldrich; Merck KGaA (Darmstadt, Germany). Sorafenib was purchased from Selleck Chemicals (Houston, TX, USA) and stored as $10 \mathrm{mM}$ stock in dimethyl sulfoxide at $-20^{\circ} \mathrm{C}$.

Determination of AFP concentrations. AFP concentrations were measured in serum samples using the Vista Dimension 500 analyser (Siemens AG, Munich, Germany) and the corresponding kit recommended for routine clinical practice. Only decreases of $>20 \%$ in the serum level of AFP were taken into consideration. This cut-off was based on the study of Personeni et al (8).

Determination of AREG and VEGF concentrations. AREG or VEGF concentrations in cell supernatant or serum samples were determined using a sandwich-based ELISA kit (R\&D Systems, Inc., Minneapolis, MN, USA) according to the manufacturer's instructions. Only $>5 \%$ decreases in the serum level of AREG or VEGF were taken into consideration This cut-off was selected as it corresponded to the smallest variation observed in our cohort.

Reverse transcription-quantitative polymerase chain reaction (RT-qPCR) analysis. Total RNA was extracted using RNeasy Mini kit (ref 74104; Qiagen, Courtaboeuf, France) and reverse-transcribed to cDNA using High Capacity cDNA Reverse Transcription kit (ref 4368814; Applied Biosystems; Thermo Fisher Scientific, Inc., Waltham, MA, USA) according to the manufacturer's instructions. Amplification was performed with the TaqMan Universal PCR master Mix (ref 4304437; Applied Biosystems; Thermo Fisher Scientific, Inc.) on an ABI 7900HT Sequence Detection System (Applied Biosystems; Thermo Fisher Scientific, Inc.) using primers and probe sets for AREG (ref Hs00950669_m1, cat. no. 433182; Applied Biosystems; Thermo Fisher Scientific, Inc.) and glyceraldehyde phosphate dehydrogenase (GAPDH; ref Hs99999905_m1, cat. no. 4333764; Applied Biosystems; Thermo Fisher Scientific, Inc.). The cycling conditions were initial denaturation at $95^{\circ} \mathrm{C}$ for $10 \mathrm{~min}$, followed by 45 cycles of denaturation at $95^{\circ} \mathrm{C}$ for $15 \mathrm{sec}$ and annealing at $60^{\circ} \mathrm{C}$ for $1 \mathrm{~min}$. Data were normalized to the endogenous control $G A P D H$ to obtain $\triangle \mathrm{Cq}$. The fold change in genes of interest relative to untreated samples was determined using the $2^{-\Delta \Delta \mathrm{Cq}}$ method (17).

Western blotting. For each experimental condition, complete cell extracts were prepared in RIPA buffer. Following protein concentration determination with a BCA kit (Thermo Fisher Scientific, Inc.), a total of $50 \mu \mathrm{g}$ protein was precipitated with methanol and chloroform. The samples were then denatured in Laemmli sample buffer, loaded on 10\% SDS-PAGE, and transferred to nitrocellulose membranes using standard procedures. The membranes were saturated for $1 \mathrm{~h}$ in $5 \%$ milk in TTBS [Tween $0.05 \%, \mathrm{NaCl} 200 \mathrm{mM}$, Tris- $\mathrm{HCl}$ (pH 8.0)], then rinsed and incubated overnight with each primary antibody at a 1:1,000 dilution (AREG, mouse, Santa Cruz Biotechnology, Inc., Dallas, TX, USA; ref 74501; and $\beta$-actin, mouse, Sigma-Aldrich; Merck KGaA; ref A544R). Later, secondary antibodies coupled with horseradish peroxidase (HRP) were incubated for $1 \mathrm{~h}$ at a 1:5,000 dilution (anti-mouse IgG HRP-conjugated, sheep, GE Healthcare, Chicago, IL, USA; ref NA931V). The electrochemiluminescence reaction was used for detection.

Cytokine array. The Proteome Profiler 'Human Cytokine Array Panel A' kit was purchased from R\&D Systems (ref ARY005) and used according to the manufacturer's instructions. Briefly, this kit utilizes capture antibodies spotted onto a nitrocellulose membrane to allow high-throughput multi-analyte profiling of 36 cytokines, chemokines and acute phase proteins in a 


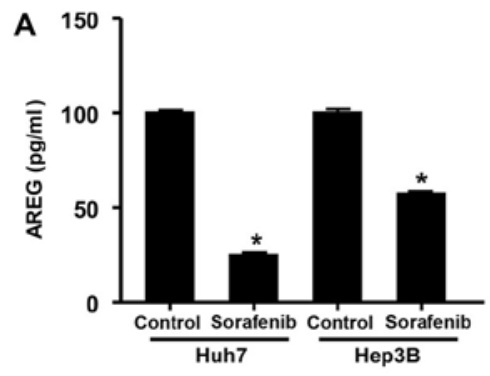

D

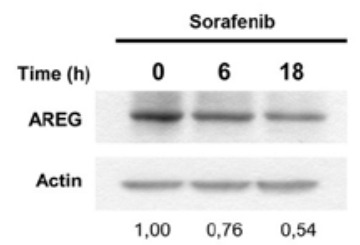

B

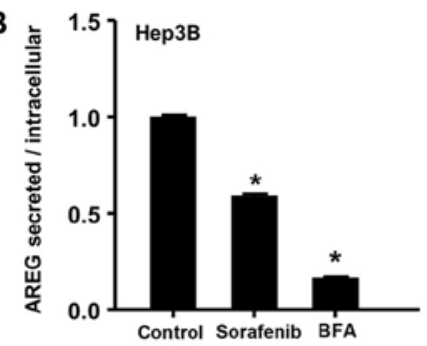

E

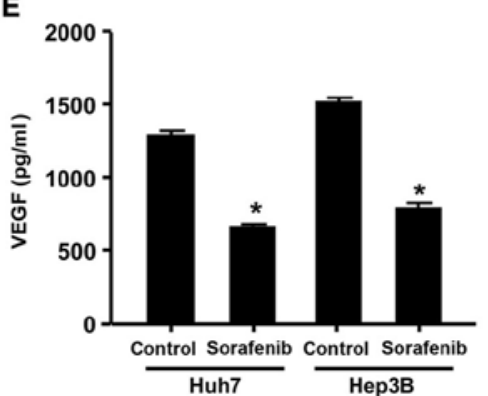

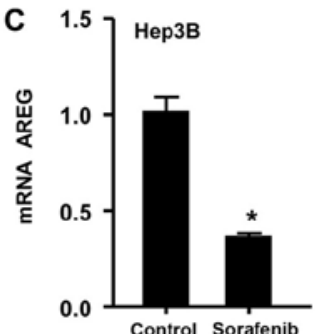

$\mathbf{F}$
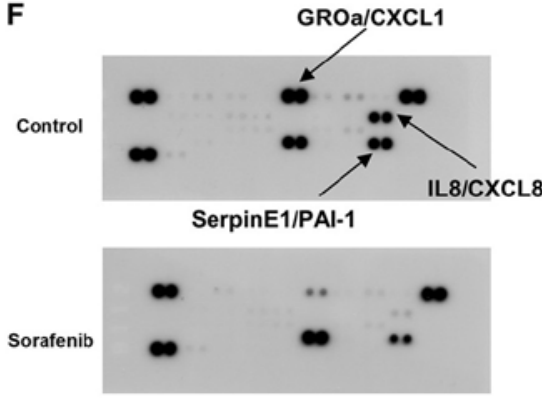

Figure 1. Sorafenib inhibits AREG, VEGF and cytokines in vitro. (A) Huh7 and Hep3B cells were treated with sorafenib (10 $\mu \mathrm{M})$ for $18 \mathrm{~h}$. The concentrations of secreted AREG were determined by ELISA in the cell supernatant. (B) Hep3B cells were treated with sorafenib (10 $\mu \mathrm{M})$ or brefeldin A (BFA; $5 \mu \mathrm{g} / \mathrm{ml})$ for $18 \mathrm{~h}$. The concentrations of secreted and intracellular AREG were determined by ELISA and the ratio was calculated after normalization with respect to control conditions. (C) qPCR analysis of the mRNA encoding AREG in Hep3B cells exposed to sorafenib (10 $\mu \mathrm{M})$ for $18 \mathrm{~h}$. (D) Protein extracts obtained from Hep3B cells exposed to $10 \mu \mathrm{M}$ sorafenib at indicated times were analysed by immunoblotting for their content of AREG or actin. The AREG/actin ratio is given for each time point, taking the control conditions as reference. (E) Huh7 and Hep3B cells were treated with sorafenib (10 $\mu \mathrm{M})$ for $18 \mathrm{~h}$. The concentrations of secreted VEGF were determined by ELISA in the cell supernatant. (F) Human cytokine array performed on Hep3B cells treated with sorafenib $(10 \mu \mathrm{M})$ for $18 \mathrm{~h}$. Experiments were performed in triplicate. ${ }^{*} \mathrm{P}<0.05$ compared with control (Student's t-test). AREG, amphiregulin; VEGF, vascular endothelial growth factor; qPCR, quantitative polymerase chain reaction.

single sample. Cell culture supernatants were diluted and mixed with a cocktail of biotinylated detection antibodies. The sample/antibody mixture was then incubated with the array. Any cytokine/antibody complex present was bound by its cognate immobilized capture antibody on the membrane. Streptavidin-HRP and chemiluminescent detection reagents were added, and a signal was produced in proportion to the amount of cytokine bound. Chemiluminescence was detected in the same manner as a western blot.

Serum samples. Frozen serum samples from a cohort of 55 patients with advanced HCC receiving sorafenib were used in this study. Patients were recruited from Cochin Hospital (Paris, France). Of the 55 patients, 50 had cancer associated with cirrhosis. The patients were treated with sorafenib at the validated dose of $400 \mathrm{mg}$ twice daily until evidence of disease progression. In 12 of the 55 patients, the dose was reduced to $50 \%$ of the planned dose due to severe drug-related adverse events (grade 3/4 hand-foot skin reaction, $n=5$; grade 3 fatigue, $\mathrm{n}=4$; and grade 3 hypertension, $\mathrm{n}=3$ ). Tumour evaluation was performed every 3 months during treatment according to the modified Response Evaluation Criteria In Solid Tumours (18). Progression-free survival (PFS) was measured from the date of diagnosis to the date of evidence of disease progression or death. OS was measured from the date of diagnosis to the date of death or the last follow-up. Serum samples were collected from each patient 7 days before and 14 days after initiating sorafenib therapy.

Ethics approval and consent to participate. The present study was conducted in compliance with the French legislation and the Declaration of Helsinki regarding ethical principles for medical research involving human subjects. The 'Comité de Protection des Personnes d'Ile de France' Institutional Review Board approved the study protocol (NuAT140) and all patients provided their written informed consent. No samples were obtained from patients who were minors or physically or mentally unable to understand and provide their consent to the use of their serum samples.

Statistical analysis. Student's t-test was used for statistical analyses of the experiments performed on cells. Wilcoxon's test, Fisher's exact test, Chi-squared test, receiver operating characteristic (ROC) curve analysis and Kaplan-Meier analysis were used as indicated for analyses performed on the patient cohort. Univariate and multivariate analyses were performed using a Cox proportional hazard regression model. $\mathrm{P}<0.05$ was considered to indicate a statistically significant difference. All analyses were performed with R, v.3.4.2 software (https://www.r-project.org).

\section{Results}

Sorafenib inhibits the production of AREG, VEGF and inflammatory cytokines by HCC cells. In order to analyse the impact of sorafenib on AREG and VEGF, two cell lines with different sensitivities to sorafenib were exposed to a clinically relevant concentration of the drug $(10 \mu \mathrm{M})$ for $18 \mathrm{~h}$. The concentration of $10 \mu \mathrm{M}$ was selected as it was close to the median concentrations measured in the serum of HCC patients by Abou-Alfa et al (19). The Huh7 cell line has been previously shown to be sensitive to sorafenib, while Hep3B 
Table I. Summary of the clinical characteristics of the patients.

Characteristics

$\begin{array}{lr}\text { Age (years, median) } & 61 \\ \text { Sex (male/female) } & 40 / 1 \\ \text { Child-Pugh class } & \\ 0 & 12 \\ \text { A } & 41 \\ \text { B } & 2\end{array}$

Main aetiology of cirrhosis

$\begin{array}{lc}\text { Alcohol } & 4 \\ \text { NASH } & 11 \\ \text { Virus } & 21 \\ \text { Mixed } & 4 \\ \text { Unknown } & 10\end{array}$

HCC, hepatocellular carcinoma; NASH, non-alcoholic steatohepatitis.

cells were found to be resistant to sorafenib in terms of clonogenic growth (15). Recently, the Huh7 and Hep3B cell lines were exposed to increasing concentrations of sorafenib $(0-20 \mu \mathrm{M})$ for $18 \mathrm{~h}$, and a decrease in cell viability of $\sim 10 \%$ was measured using the Trypan blue exclusion assay (9). This modest cytotoxic effect is essentially accounted for by ferroptosis, a form of necrotic cell death (20).

A significant decrease in AREG concentrations in the cell supernatant was observed following sorafenib exposure in both cell lines (Fig. 1A). In order to examine the relative contribution of defective production vs. secretion of AREG, the concentration of this protein was measured in cell supernatants and cell lysates following exposure of Hep3B cells to sorafenib. The secretion blocker brefeldin A was used as positive control. The ratio of secreted to intracellular AREG was calculated under control conditions or after exposure to sorafenib or brefeldin A. Following sorafenib exposure, this ratio was calculated to be 0.60 , suggesting that the inhibitory effect of sorafenib on AREG resulted from both inhibition of AREG production (60\%) and inhibition of AREG secretion (40\%) (Fig. 1B). In order to confirm the effect of sorafenib on AREG production, the effect of sorafenib on AREG mRNA levels was examined and AREG protein levels were determined by western blot analysis using Hep3B cells. An inhibition of $65 \%$ in AREG mRNA expression was observed following exposure to sorafenib (Fig. 1C). A decrease of $~ 50 \%$ in AREG protein levels was measured by western blot analysis (Fig. 1D). A significant decrease of VEGF levels in the cell supernatant was also observed following sorafenib exposure in both cell lines (Fig. 1E). Finally, in order to explore the effect of sorafenib on several cytokines, a cytokine array was performed. Inhibition of three cytokines, namely CXCL1, IL8 and SERPIN E1, was observed (Fig. 1F). Sorafenib exerted an inhibitory effect on AREG, VEGF and cytokine expression in vitro at the transcriptional and post-transcriptional level, suggesting that sorafenib induced a broad inhibition of bioactive protein production.
Prognostic role of AFP, AREG and VEGF in HCC patients prior to sorafenib treatment. The clinical characteristics of the patients are summarized in Table I and are also detailed in a previous study (21). The median OS assessed by the Kaplan-Meier method was 23.6 months for all 55 patients enrolled in this study. AFP concentrations were determined in the serum samples of 38 patients among the cohort of 55 patients, and the concentrations in 19 patients were undetectable $(<10 \mathrm{ng} / \mathrm{ml})$. By contrast, detectable concentrations of AREG and VEGF were measured in the 55 patients of our cohort. In order to evaluate the prognostic value of AFP, AREG and VEGF prior to treatment, patients were separated according to their baseline AFP, AREG and VEGF concentrations as below or above the median. Kaplan-Meier analysis of OS demonstrated that baseline AFP, AREG or VEGF concentrations prior to sorafenib therapy did not constitute a discriminant prognostic biomarker (Fig. 2A-C; $\mathrm{P}=0.10, \mathrm{P}=0.98$ and $\mathrm{P}=0.91$, respectively). A marked interand intra-individual heterogeneity was observed between AFP, AREG and VEGF levels before and after sorafenib treatment (data not shown). Among the 19 patients with detectable concentrations of AFP, pretreatment AFP concentrations were not significantly different from the concentrations obtained after 2 weeks of sorafenib therapy $(2,581 \pm 1,053$ vs. $1,935 \pm 740 \mathrm{ng} / \mathrm{ml} ; \mathrm{P}=0.62)$. Similarly, among the 55 patients of the cohort, pretreatment AREG and VEGF concentrations were not significantly different from the concentrations obtained after 2 weeks of sorafenib therapy $(60.6 \pm 103$ vs. $59.6 \pm 105 \mathrm{pg} / \mathrm{ml}, \mathrm{P}=0.91$ for $\mathrm{AREG}$; and $516.6 \pm 51$ vs. $495.0 \pm 43 \mathrm{pg} / \mathrm{ml}, \mathrm{P}=0.75$ for VEGF).

Prognostic role of a decrease in serum AFP levels. Among the 19 patients with detectable concentrations of AFP, we observed a significant decrease in AFP (>20\%) after sorafenib treatment in only 3 patients. OS and PFS were evaluated in patients with a decrease in AFP levels vs. patients with no decrease after 2 weeks of sorafenib therapy. The median survival was 31.7 months in patients with a decrease in AFP $(n=3)$ vs. 21.3 months in the group with no decrease in AFP levels $(\mathrm{n}=16)$. The difference between these two groups was not significant (Fig. 2D, P=0.20). The PFS in patients with a decrease in AFP was not significantly different from that in patients with no decrease in AFP levels (Fig 2E, median 27 vs. 18 months; $\mathrm{P}=0.22)$.

A decrease in serum AREG or VEGF levels predicts sorafenib efficacy. In order to evaluate the prognostic value of intra-individual variations of AREG and VEGF, patients were classified according to their VEGF or AREG variations following sorafenib therapy. No significant difference was observed between these groups (presented in Table II for AREG and Table III for VEGF). OS and PFS were evaluated in patients with a decrease in one of these biomarkers $(>5 \%)$ vs. patients with no decrease, after 2 weeks of sorafenib therapy. The median survival was 38.9 months in patients with a decrease in AREG ( $n=26)$ vs. 15.8 months in the group with no decrease in AREG $(n=29)$. The difference between these two groups was statistically significant (Fig. 2F; $P=0.0009$ ). A statistically significant difference in PFS was also observed between these two groups (Fig. 2G, median 46.9 vs. 21.1 months; $\mathrm{P}=0.0014$ ). 
A
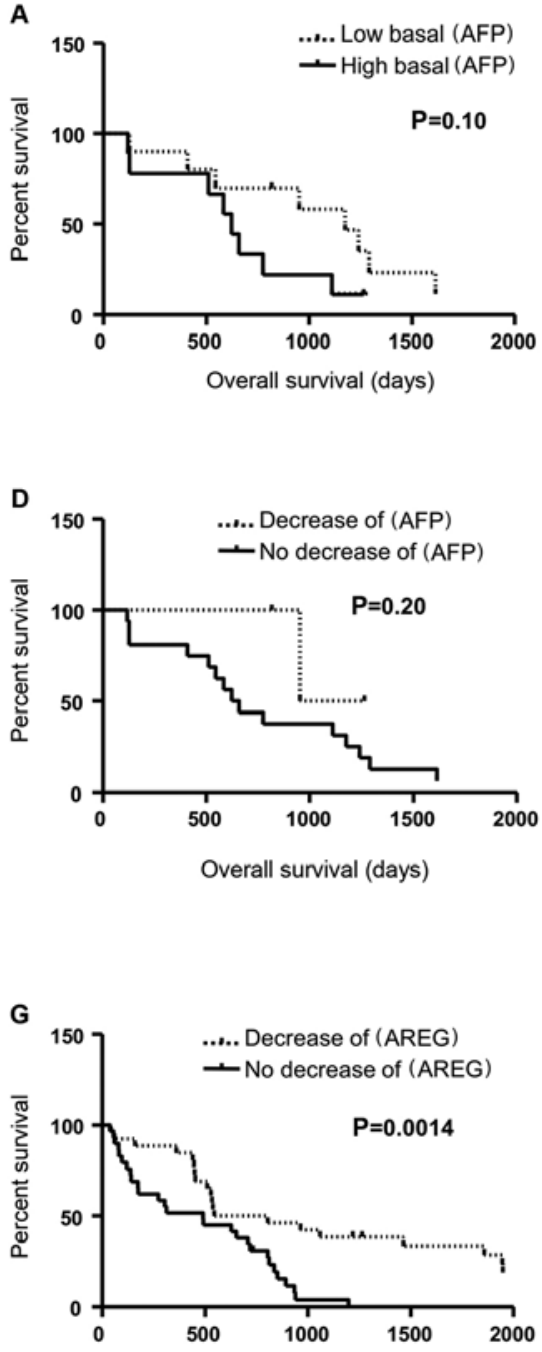

Progression-free survival (days)
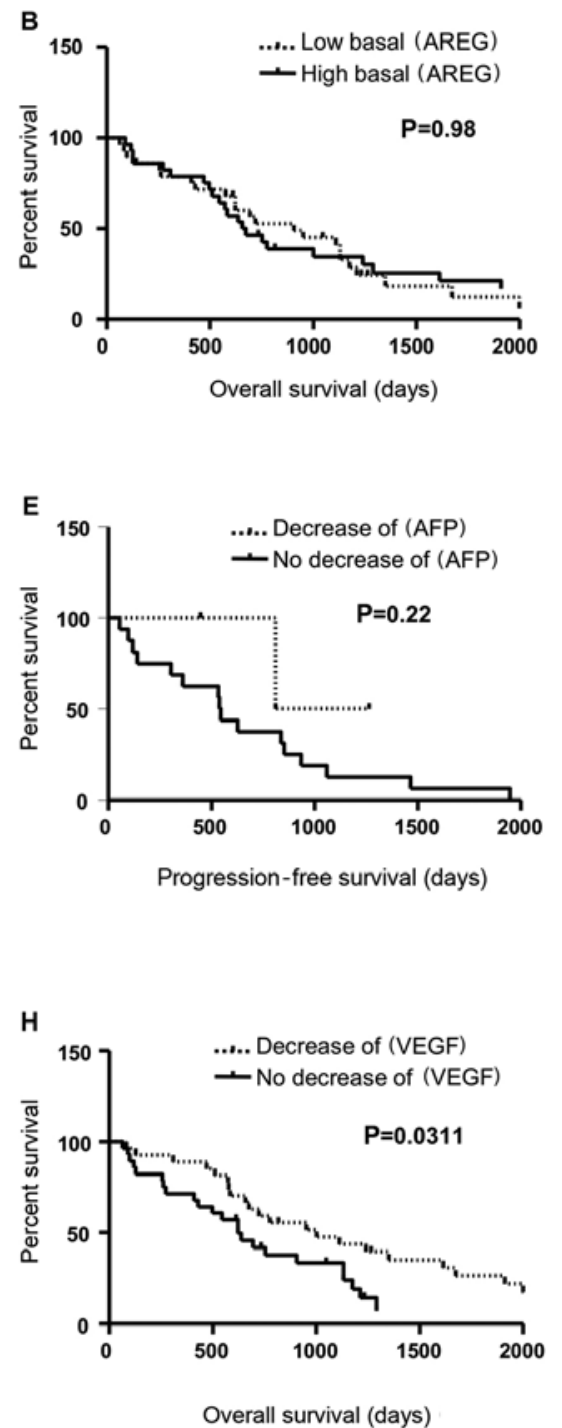
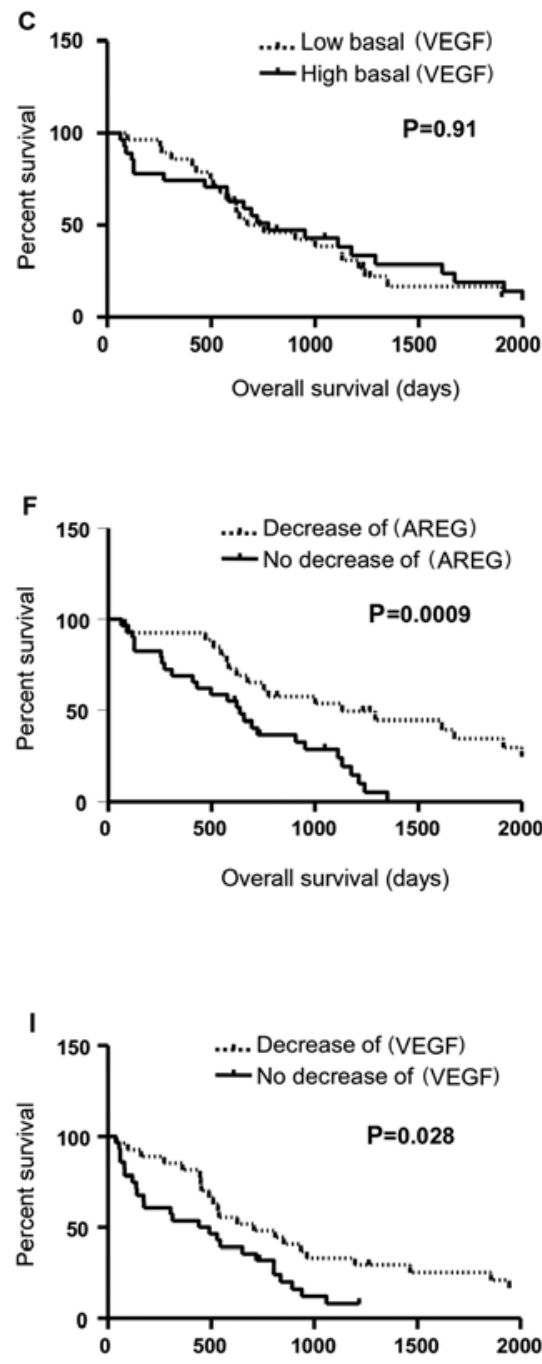

Progression-free survival (days)

Figure 2. Prognostic role of AFP, AREG and VEGF in HCC patients treated by sorafenib. (A-C) Kaplan-Meier analysis of OS of patients divided into two groups according to their baseline (A) AFP, (B) AREG of (C) VEGF levels (below or above the median). (D) Kaplan-Meier analysis of the OS of patients divided into two groups according to their variations in AFP before and after sorafenib therapy (presence or absence of a $\geq 20 \%$ decrease of AFP after treatment). (E) Kaplan-Meier analysis of the PFS of patients divided into two groups according to their variations in AFP before and after sorafenib therapy (presence or absence of $\mathrm{a} \geq 20 \%$ decrease of AFP after treatment). (F) Kaplan-Meier analysis of the OS of patients divided into two groups according to their variations of AREG before and after sorafenib therapy (presence or absence of a $\geq 5 \%$ decrease of AREG after treatment). (G) Kaplan-Meier analysis of the PFS of patients divided into two groups according to their variations of AREG before and after sorafenib therapy (presence or absence of a $\geq 5 \%$ decrease of AREG after treatment). (H) Kaplan-Meier analysis of the OS of patients divided into two groups according to their variations of VEGF before and after sorafenib therapy (presence or absence of a $\geq 5 \%$ decrease of VEGF after treatment). (I) Kaplan-Meier analysis of PFS of patients divided into two groups according to their variations of VEGF before and after sorafenib therapy (presence or absence of a $\geq 5 \%$ decrease of VEGF after treatment). AFP, $\alpha$-fetoprotein; AREG, amphiregulin; VEGF, vascular endothelial growth factor; HCC, hepatocellular carcinoma; PFS, progression-free survival; OS, overall survival.

Interestingly, the group of patients with no decrease in AREG was composed of 1 patient with a stable level of AREG and 28 patients with an increase in AREG levels of at least $20 \%$ after 15 days of sorafenib treatment. Therefore, as shown in Fig. 2F and G, an increase in AREG levels was correlated with a poor prognosis.

For VEGF, the median survival was 27.2 months in patients with a decrease in VEGF $(n=23)$ vs. 22.2 months in the group with no decrease in VEGF $(n=32)$. A significant difference in OS was observed between these two groups (Fig. $2 \mathrm{H}$; $\mathrm{P}=0.0311$ ). A significant difference was also observed in PFS (Fig. 2I, median 20.9 vs. 16.9 months; $\mathrm{P}=0.028$ ). The prognostic value of each clinicopathological factor, including AREG decrease, VEGF decrease, age, sex, Child-Pugh score and cirrhosis aetiology, was evaluated by univariate and multivariate analysis for OS (Table IV). Univariate analysis indicated that a decrease in AREG levels was significantly associated with $\mathrm{OS}(\mathrm{HR}=0.341 ; \mathrm{P}=0.002)$. Further multivariate analysis demonstrated that a decrease in AREG levels could be considered as an independent prognostic biomarker for OS $(\mathrm{HR}=0.208 ; \mathrm{P}=0.00034 ; 95 \% \mathrm{CI}: 0.173-0.673)$.

In order to confirm these results, the clinical usefulness of a decrease in AREG or VEGF for prediction of OS status at 1, 3 and 5 years was evaluated by time-dependent ROC curve analyses. A decrease in AREG was predictive for OS at 5 years, with an area under the curve (AUC) of 0.77 . The AUC was 0.68 at 1 year. For VEGF, AUCs of 0.67 and 0.57 were obtained for OS at 5 years and at 1 year, respectively (Fig. 3). 
Table II. Summary of the clinical characteristics of the patients categorized according to their variation in serum levels of AREG after 14 days of sorafenib treatment.

\begin{tabular}{lccc}
\hline Characteristics & $\begin{array}{c}\text { Decrease of AREG } \\
(\mathrm{n}=29)\end{array}$ & $\begin{array}{c}\text { Decrease of AREG } \\
(\mathrm{n}=26)\end{array}$ & \begin{tabular}{c} 
P-value \\
\hline Age (years, median)
\end{tabular} \\
Sex (male/female) & 61 & 61 & $0.97^{\mathrm{a}}$ \\
Child-Pugh class & $20 / 9$ & $20 / 6$ & $0.72^{\mathrm{b}}$ \\
0 & & & $0.35^{\mathrm{c}}$ \\
A & 6 & 6 & $0.16^{\mathrm{c}}$ \\
B & 23 & 18 & \\
Main aetiology of cirrhosis & 0 & 2 & \\
Alcohol & & 4 & \\
NASH & 0 & 5 & \\
Virus & 6 & 10 & \\
Mixed & 11 & 4 & \\
Unknown & 3 & 6 & \\
\hline
\end{tabular}

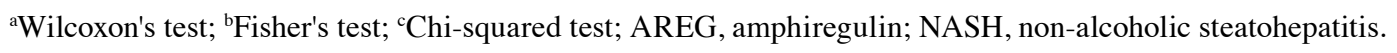

Table III. Summary of the clinical characteristics of patients categorized according to their variation in serum levels of VEGF after 14 days of sorafenib treatment.

\begin{tabular}{lccc}
\hline Characteristics & $\begin{array}{c}\text { No decrease of VEGF } \\
(\mathrm{n}=32)\end{array}$ & $\begin{array}{c}\text { Decrease of VEGF } \\
(\mathrm{n}=23)\end{array}$ & P-value \\
\hline Age (years, median) & 61 & 62 & $0.76^{\mathrm{a}}$ \\
Sex (male/female) & $21 / 11$ & $19 / 4$ & $0.22^{\mathrm{b}}$ \\
Child-Pugh class & & & $0.77^{\mathrm{c}}$ \\
0 & 6 & 6 & $0.73^{\mathrm{c}}$ \\
A & 25 & 16 & \\
B & 1 & 1 & \\
Main aetiology of cirrhosis & & 2 & \\
Alcohol & 2 & 5 & \\
NASH & 6 & 8 & \\
Virus & 13 & 3 & \\
Mixed & 1 & 3 & \\
Unknown & 7 & & \\
\hline
\end{tabular}

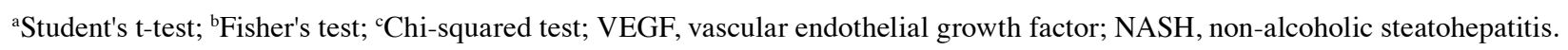

The regulation of AREG and VEGF by sorafenib is potentially mediated via a common pathway. To investigate the hypothesis of a common pathway regulating AREG and VEGF, the prognostic value of variations of both AREG and VEGF were evaluated. The median OS assessed by the Kaplan-Meier method in patients with a decrease in both AREG and VEGF was 40.5 vs. 21.9 months in the group with an increase or discordant results for these two markers (Fig. 4A; $\mathrm{P}=0.0085$ ). A significant difference was also observed in PFS (median 23.6 vs. 17 months, $\mathrm{P}=0.017$; Fig. $4 \mathrm{~B}$ ). These results suggest that the combination of these two biomarkers was not more discriminant in terms of prognosis compared with each biomarker alone. The variations of these biomarkers were compared in each patient. Interestingly, the two markers varied in the same direction (decrease or increase) in $69 \%$ of the patients ( $n=38)$ (Fig. 4C). A Chi-squared test revealed that the variations of these two biomarkers were dependent (Fig. 4D; $\mathrm{P}=0.007)$, suggesting that AREG and VEGF are regulated by sorafenib via a common mechanism.

\section{Discussion}

The present study demonstrated that sorafenib decreased AREG, VEGF and cytokine levels in HCC cells at the 
Table IV. Univariate and multivariate analyses of overall survival in HCC patients.

\begin{tabular}{|c|c|c|c|c|c|c|}
\hline \multirow[b]{2}{*}{ Variables } & \multicolumn{3}{|c|}{ Univariate analysis } & \multicolumn{3}{|c|}{ Multivariate analysis } \\
\hline & HR 95\% & $\mathrm{CI}$ & P-value & HR & $95 \% \mathrm{CI}$ & P-value \\
\hline Age (years) & 1.013 & $0.989-1.038$ & 0.291 & 1.006 & $0.978-1.035$ & 0.683 \\
\hline Sex (male) & 1.323 & $0.690-2.538$ & 0.399 & 1.342 & $0.642-2.807$ & 0.434 \\
\hline Child-Pugh A & 1.162 & $0.556-2.427$ & 0.690 & 0.423 & $0.061-2.938$ & 0.384 \\
\hline Child-Pugh B & 7.968 & $1.565-40.560$ & 0.012 & 6.681 & $0.494-90.398$ & 0.153 \\
\hline Cirrhosis & 1.170 & $0.453-3.017$ & 0.746 & 1.295 & $0.109-15.351$ & 0.838 \\
\hline \multicolumn{7}{|c|}{ Aetiology of cirrhosis } \\
\hline Alcohol & 3.287 & $0.834-12.948$ & 0.089 & 8.405 & $1.606-43.997$ & 0.012 \\
\hline Virus & 1.318 & $0.481-3.610$ & 0.592 & 1.583 & $0.478-5.239$ & 0.452 \\
\hline NASH & 0.700 & $0.231-2.124$ & 0.529 & 1.008 & $0.255-3.990$ & 0.991 \\
\hline Mixed & 1.913 & $0.507-7.223$ & 0.339 & NA & NA & NA \\
\hline AREG decrease & 0.341 & $0.173-0.673$ & 0.002 & 0.208 & $0.088-0.491$ & 0.0003 \\
\hline VEGF decrease & 0.583 & $0.312-1.092$ & 0.092 & 0.720 & $0.360-1.438$ & 0.352 \\
\hline
\end{tabular}

Bold print indicates statistical significance. HCC, hepatocellular carcinoma; HR, hazard ratio estimated from Cox proportional hazard regression model; CI, confidence interval of the estimated HR; NASH, non-alcoholic steatohepatitis; AREG, amphiregulin; VEGF, vascular endothelial growth factor; NA, not available.
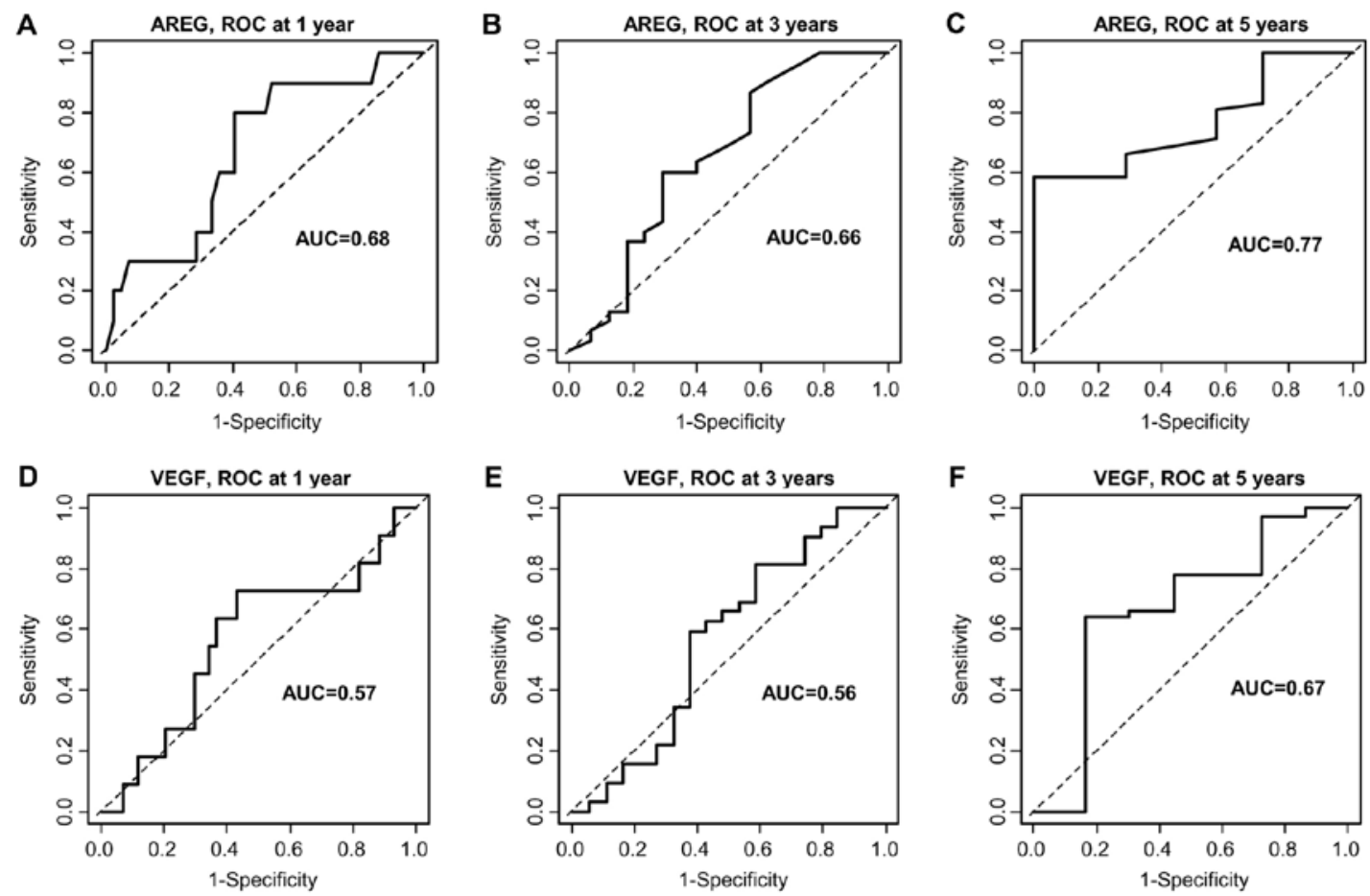

Figure 3. Prognostic value of a decrease in AREG or VEGF evaluated by time-dependent ROC curve analyses. (A-C) ROC curve analyses for prediction of OS status at 1,3 and 5 years for AREG decrease. (D-F) ROC curve analyses for prediction of OS status at 1, 3 and 5 years for VEGF decrease. AREG, amphiregulin; VEGF, vascular endothelial growth factor; ROC, receiver operating characteristics; OS, overall survival.

transcriptional and post-transcriptional levels. These results suggest that sorafenib induced an inhibition of the production of biologically active proteins, in accordance with our recent study highlighting a global inhibitory effect of sorafenib on protein biosynthesis (9). This previous study was performed in vitro and, therefore, did not explore the consequences of this inhibition in a clinical setting. In the present study, a decrease in serum AREG and VEGF levels was identified as a potential biomarker of sorafenib efficacy in HCC patients.

Sorafenib is the standard of care for the medical treatment of advanced-stage HCC, but there is a lack of biomarkers to evaluate its effectiveness. AFP is the serum biomarker most 

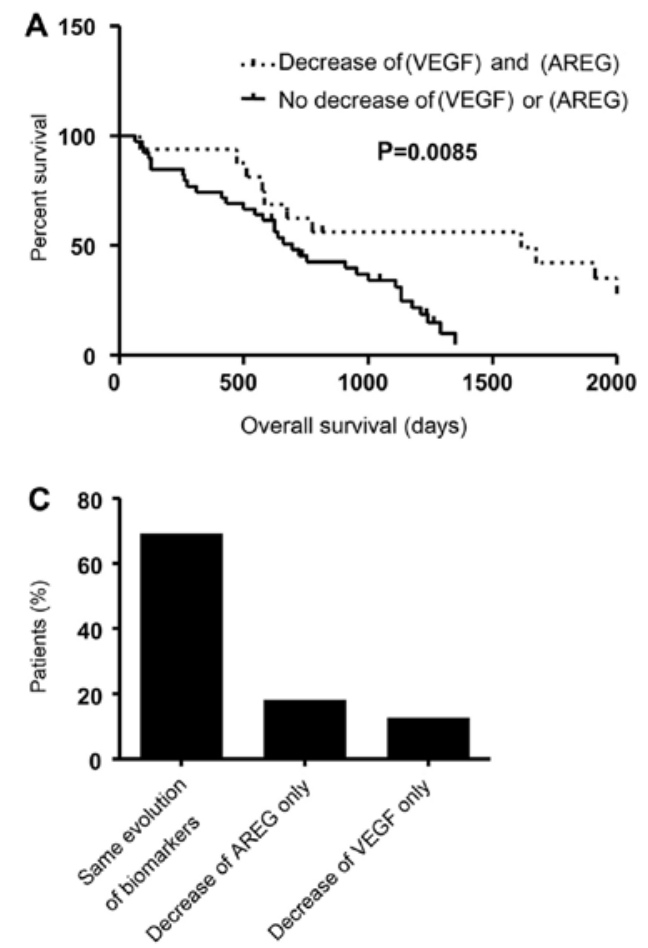

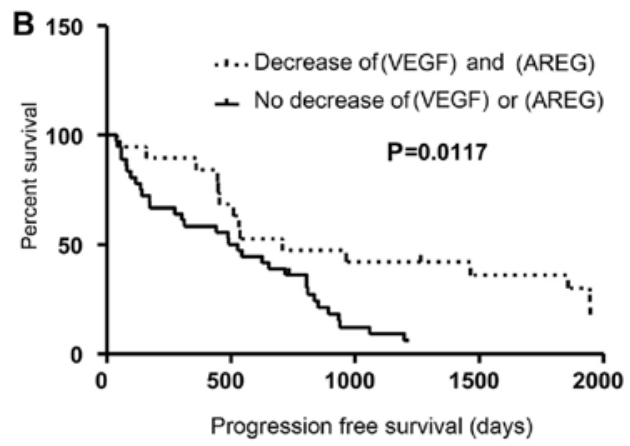

D

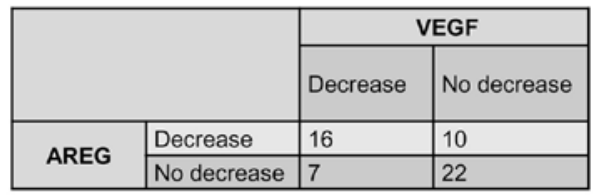

$\mathrm{P}=0.007$

Figure 4. Variations of AREG and VEGF levels are correlated in HCC patient survival. (A) Kaplan-Meier analysis of the OS of patients with a decrease of both AREG and VEGF levels compared with the other patients. (B) Kaplan-Meier analysis of the PFS of patients with a decrease of both AREG and VEGF compared with the other patients. (C and D) Comparison of the concordance of the direction of the variations of AREG and VEGF concentrations in patients. AREG, amphiregulin; VEGF, vascular endothelial growth factor; HCC, hepatocellular carcinoma; OS, overall survival; PFS, progression-free survival.

commonly used by clinicians to evaluate sorafenib efficacy. It was previously reported that a decrease of $20 \%$ in the serum levels of AFP measured during the first 8 weeks of sorafenib treatment was associated with better OS, while imaging was not predictive in this context (8). However, a significant decrease in serum AFP levels following sorafenib treatment is observed in only $\sim 18 \%$ of the patients (8). In our cohort, we observed such a decrease in only 3 of 38 patients. Due to the small number of patients, a decrease in serum levels of AFP was not found to be associated with a statistically significant improvement in OS or PFS in our cohort (Fig. 2D and $\mathrm{E}, \mathrm{P}=0.20$ and $\mathrm{P}=0.22$, respectively). These results confirm that the clinical value of AFP is limited to a small number of patients.

In the present study, a decrease in serum AREG or VEGF levels after only 15 days of sorafenib treatment was shown to predict sorafenib efficacy. In particular, Kaplan-Meier analyses revealed that a decrease in serum AREG or VEGF levels after 2 weeks of sorafenib treatment was associated with a significantly better OS and PFS. A difference was observed for VEGF and AREG in the multivariate analysis. A decrease in AREG, but not in VEGF, was a significant prognostic biomarker for OS. This difference was also observed with the ROC curve analyses. A decrease in AREG was predictive of OS at 5 years with an AUC of 0.77, whereas the AUC was 0.67 for VEGF. Based on these results, we propose that a decrease in both serum VEGF and AREG levels may be considered as a prognostic biomarker, but it is likely that the decrease in serum AREG levels is a better biomarker in this context. Unlike AFP, AREG and VEGF can be detected in the serum of all HCC patients. The finding that their early decrease after sorafenib treatment was correlated with the efficacy of sorafenib may be of interest to clinicians.
These biomarkers may help clinicians identify patients for whom sorafenib is not expected to be beneficial and to consider therapeutic alternatives at an earlier stage. A prospective study comparing the prognostic role of AFP, AREG and VEGF on a larger number of patients is required to confirm these results.

Previous studies have investigated the prognostic role of AREG and VEGF in HCC patients. Our conclusions regarding VEGF confirm and extend those of another previous study by Tsuchiya $e t$ al. In complete agreement with our results, these authors observed that a decrease in VEGF after 8 weeks of sorafenib therapy was associated with better OS on Kaplan-Meier analysis (22). Three studies have already explored AREG as a potential prognostic biomarker in HCC patients, two of which evaluated AREG at baseline and one after sorafenib treatment. In agreement with the two studies evaluating AREG at baseline, we did not observe any correlation between pretreatment AREG levels and prognosis $(23,24)$. In the present study, although large fluctuations were observed in AREG concentrations in patients, pretreatment AREG concentrations were not statistically significantly different from the concentrations measured after 2 weeks of sorafenib therapy (Fig. 2A). This may be explained by the marked inter- and intra-individual heterogeneity in AREG levels before and after sorafenib treatment. The study by Blivet-Van Eggelpöel et al is the only study to have investigated variations in serum AREG levels before and after sorafenib therapy. In contrast with the present study, these authors demonstrated that serum AREG levels were markedly increased in HCC patients treated with sorafenib compared with baseline levels. This study was conducted on an exploratory cohort of only 14 patients, and did not evaluate the prognostic role of 
AREG (16). Blivet-Van Eggelpöel et al observed an increase in serum concentrations of AREG in 10 out of 14 patients receiving sorafenib. The authors indicated that "no complete or partial tumour response to sorafenib was present in these patients. Six of them showed disease progression'. Our results are consistent with theirs, indicating that an increase in AREG is not in favour of sorafenib efficacy. In the Blivet-Van Eggelpöel et al study, serum AREG levels were measured after 28-458 days of sorafenib treatment. Unfortunately, the study did not provide additional dosages beyond 15 days. A complementary study would be of interest.

Apart from the prognostic role of AREG and VEGF in HCC patients treated by sorafenib, the results of the present study suggest that these two biomarkers are regulated by a common mechanism, as a statistically significant correlation was observed between variations in serum AREG and VEGF levels. The concentrations of these two biomarkers varied in the same direction (increase or decrease) in $69 \%$ of the patients after sorafenib therapy. The combination of these two biomarkers was not a more discriminant predictive factor of the efficacy of sorafenib compared with each biomarker alone. This potential common pathway of regulation of AREG and VEGF may be associated with the effect of sorafenib on tumour proteostasis. Sorafenib alters tumour proteostasis in a complex way and via several different mechanisms $(9,20,25-27)$. Tumour proteostasis includes emerging drivers of tumour progression and important determinants of clinical efficacy of cancer therapy (28). Our recent findings indicate that tumour markers, such as AFP, are regulated by tumour proteostasis $(29,30)$. It is hypothesized that the decrease of AREG and VEGF production may be associated with sorafenib-induced alteration of proteostasis. A thorough exploration of the role of proteostasis as a regulator of tumour response to sorafenib is required.

In conclusion, the present study demonstrated a broad inhibitory effect of sorafenib on the production of bioactive proteins, such as AREG and VEGF, in vitro. The decrease of serum AREG or VEGF levels was found to be associated with better OS and PFS in HCC patients. At this stage, the role of sorafenib in these decreases remains to be formally demonstrated. However, this effect may be associated with the ability of sorafenib to alter proteostasis, as shown in a previous study. The present study identified two biomarkers of sorafenib efficacy, detectable early in all HCC patients, and potentially of interest to clinicians.

\section{Acknowledgements}

The authors would like to thank Momar Diouf for his help with ROC curve analyses.

\section{Funding}

The authors are grateful for the financial support received from 'Ligue Contre le Cancer', 'Conseil Régional des Hauts de France (Soramix)', 'Comité de la Somme' and 'CHU Amiens'.

\section{Availability of data and materials}

The datasets used and/or analysed during the present study are available from the corresponding author on reasonable request.

\section{Authors' contributions}

RC, AG and CS contributed to the study design. CG, SB, ZS, CL and CS collected the data. CL, JCB, CG, CF, RC, AG and CS analyzed and interpreted the data. CS drafted the manuscript. SB, ZS, JCB, RC and AG revised the manuscript. All authors have read and approved the final version of the manuscript.

\section{Ethics approval and consent to participate}

This study was conducted in compliance with French legislation and the Declaration of Helsinki regarding ethical principles for medical research involving human subjects. The 'Comité de Protection des Personnes d'Ile de France' Institutional Review Board approved the study protocol (Nu-AT140). All patients provided their written informed consent. No samples were obtained from patients who were minors or physically or mentally unable to understand and provide their consent to the use of serum samples.

\section{Patient consent for publication}

Not applicable.

\section{Competing interests}

JCB received honoraria as a clinical consultant from Bayer. Bayer was not involved in this study. Bayer did not provide any reagent and did not contribute to the design of this study. All other authors declare that they have no competing interests.

\section{References}

1. European Association for the Study of the Liver. Electronic address: easloffice@easloffice.eu, European Association for the Study of the Liver: EASL Clinical Practice Guidelines: Management of hepatocellular carcinoma. J Hepatol 69: 182-236, 2018.

2. Davis MI, Hunt JP, Herrgard S, Ciceri P, Wodicka LM, Pallares G, Hocker M, Treiber DK and Zarrinkar PP: Comprehensive analysis of kinase inhibitor selectivity. Nat Biotechnol 29: 1046-1051, 2011

3. Llovet JM, Ricci S, Mazzaferro V, Hilgard P, Gane E, Blanc JF, de Oliveira AC, Santoro A, Raoul JL, Forner A, et al; SHARP Investigators Study Group: Sorafenib in advanced hepatocellular carcinoma. N Engl J Med 359: 378-390, 2008.

4. Cheng AL, Kang YK, Chen Z, Tsao CJ, Qin S, Kim JS, Luo R, Feng J, Ye S, Yang TS, et al: Efficacy and safety of sorafenib in patients in the Asia-Pacific region with advanced hepatocellular carcinoma: A phase III randomised, double-blind, placebo-controlled trial. Lancet Oncol 10: 25-34, 2009.

5. Kudo M, Finn RS, Qin S, Han KH, Ikeda K, Piscaglia F, Baron A, Park JW, Han G, Jassem J, et al: Lenvatinib versus sorafenib in first-line treatment of patients with unresectable hepatocellular carcinoma: A randomised phase 3 non-inferiority trial. Lancet 391: 1163-1173, 2018.

6. Sauzay C, Petit A, Bourgeois AM, Barbare JC, Chauffert B, Galmiche A and Houessinon A: Alpha-foetoprotein (AFP): A multi-purpose marker in hepatocellular carcinoma. Clin Chim Acta 463: 39-44, 2016

7. Bruix J, Reig M and Sherman M: Evidence-based diagnosis, staging, and treatment of patients with hepatocellular carcinoma. Gastroenterology 150: 835-853, 2016.

8. Personeni N, Bozzarelli S, Pressiani T, Rimassa L, Tronconi MC, Sclafani F, Carnaghi C, Pedicini V, Giordano L and Santoro A: Usefulness of alpha-fetoprotein response in patients treated with sorafenib for advanced hepatocellular carcinoma. J Hepatol 57: 101-107, 2012. 
9. Sauzay C, Louandre C, Bodeau S, Anglade F, Godin C, Saidak Z, Fontaine JX, Usureau C, Martin N, Molinie R, et al: Protein biosynthesis, a target of sorafenib, interferes with the unfolded protein response (UPR) and ferroptosis in hepatocellular carcinoma cells. Oncotarget 9: 8400-8414, 2018.

10. Manié SN, Lebeau J and Chevet E: Cellular mechanisms of endoplasmic reticulum stress signaling in health and disease. 3. Orchestrating the unfolded protein response in oncogenesis: An update. Am J Physiol Cell Physiol 307: C901-C907, 2014.

11. Raskopf E, Vogt A, Sauerbruch T and Schmitz V: siRNA targeting VEGF inhibits hepatocellular carcinoma growth and tumor angiogenesis in vivo. J Hepatol 49: 977-984, 2008.

12. Goel HL and Mercurio AM: VEGF targets the tumour cell. Nat Rev Cancer 13: 871-882, 2013.

13. Castillo J, Erroba E, Perugorría MJ, Santamaría M, Lee DC, Prieto J, Avila MA and Berasain C: Amphiregulin contributes to the transformed phenotype of human hepatocellular carcinoma cells. Cancer Res 66: 6129-6138, 2006.

14. Xu Q, Chiao P and Sun Y: Amphiregulin in Cancer: New Insights for Translational Medicine. Trends Cancer 2: 111-113, 2016.

15. Ezzoukhry Z, Louandre C, Trécherel E, Godin C, Chauffert B, Dupont S, Diouf M, Barbare JC, Mazière JC and Galmiche A: EGFR activation is a potential determinant of primary resistance of hepatocellular carcinoma cells to sorafenib. Int J Cancer 131: 2961-2969, 2012

16. Blivet-Van Eggelpoël MJ, Chettouh H, Fartoux L, Aoudjehane L, Barbu V, Rey C, Priam S, Housset C, Rosmorduc O and Desbois-Mouthon C: Epidermal growth factor receptor and HER-3 restrict cell response to sorafenib in hepatocellular carcinoma cells. J Hepatol 57: 108-115, 2012.

17. Livak KJ and Schmittgen TD: Analysis of relative gene expression data using real-time quantitative PCR and the 2(-Delta Delta C(T)) Method. Methods 25: 402-408, 2001.

18. Lencioni R: New data supporting modified RECIST (mRECIST) for Hepatocellular Carcinoma. Clin Cancer Res 19: 1312-1314, 2013.

19. Abou-Alfa GK, Schwartz L, Ricci S, Amadori D, Santoro A, Figer A, De Greve J, Douillard JY, Lathia C, Schwartz B, et al: Phase II study of sorafenib in patients with advanced hepatocellular carcinoma. J Clin Oncol 24: 4293-4300, 2006.

20. Louandre C, Ezzoukhry Z, Godin C, Barbare JC, Mazière JC, Chauffert B and Galmiche A: Iron-dependent cell death of hepatocellular carcinoma cells exposed to sorafenib. Int J Cancer 133 : 1732-1742, 2013
21. Houessinon A, François C, Sauzay C, Louandre C, Mongelard G, Godin C, Bodeau S, Takahashi S, Saidak Z, Gutierrez L, et al: Metallothionein-1 as a biomarker of altered redox metabolism in hepatocellular carcinoma cells exposed to sorafenib. Mol Cancer 15: 38, 2016.

22. Tsuchiya K, Asahina Y, Matsuda S, Muraoka M, Nakata T, Suzuki Y, Tamaki N, Yasui Y, Suzuki S, Hosokawa T, et al: Changes in plasma vascular endothelial growth factor at 8 weeks after sorafenib administration as predictors of survival for advanced hepato-cellular carcinoma. Cancer 120: 229-237, 2014.

23. Han SX, Bai E, Jin GH, He CC, Guo XJ, Wang LJ, Li M, Ying X and Zhu Q: Expression and clinical significance of YAP, TAZ, and AREG in hepatocellular carcinoma. J Immunol Res 2014: 261365, 2014

24. Zhu AX, Kang YK, Rosmorduc O, Evans TR, Santoro A, Ross P, Gane E, Vogel A, Jeffers M, Meinhardt G, et al: Biomarker analyses of clinical outcomes in patients with advanced hepatocellular carcinoma treated with sorafenib with or without erlotinib in the SEARCH trial. Clin Cancer Res 22: 4870-4879, 2016.

25. Booth L, Shuch B, Albers T, Roberts JL, Tavallai M, Proniuk S, Zukiwski A, Wang D, Chen CS, Bottaro D, et al: Multi-kinase inhibitors can associate with heat shock proteins through their NH2-termini by which they suppress chaperone function. Oncotarget 7: 12975-12996, 2016.

26. Shi YH, Ding ZB, Zhou J, Hui B, Shi GM, Ke AW, Wang XY, Dai Z, Peng YF, Gu CY, et al: Targeting autophagy enhances sorafenib lethality for hepatocellular carcinoma via ER stress-related apoptosis. Autophagy 7: 1159-1172, 2011.

27. Shimizu S, Takehara T, Hikita H, Kodama T, Tsunematsu H, Miyagi T, Hosui A, Ishida H, Tatsumi T, Kanto T, et al: Inhibition of autophagy potentiates the antitumor effect of the multikinase inhibitor sorafenib in hepatocellular carcinoma. Int J Cancer 131: 548-557, 2012.

28. Hetz C, Chevet E and Oakes SA: Erratum: Proteostasis control by the unfolded protein response. Nat Cell Biol 17: 829-838, 2015.

29. Galmiche A, Sauzay C, Houessinon A, Chauffert B and Pluquet O: Probing Tumour Proteostasis and the UPR with Serum Markers. Trends Cancer 2: 219-221, 2016.

30. Houessinon A, Gicquel A, Bochereau F, Louandre C, Nyga R, Godin C, Degonville J, Fournier E, Saidak Z, Drullion C, et al: Alpha-fetoprotein is a biomarker of unfolded protein response and altered proteostasis in hepatocellular carcinoma cells exposed to sorafenib. Cancer Lett 370: 242-249, 2016. 